\title{
Adult Intestinal Botulism
}

National Cancer Institute

\section{Source}

National Cancer Institute. Adult Intestinal Botulism. NCI Thesaurus. Code C128344.

A rare form of botulism that occurs among adults by the same mechanism as infant botulism. 